\title{
OPEN PACKING NUMBER FOR SOME CLASSES OF PERFECT GRAPHS
}

\author{
K. Raja Chandrasekar \\ Amrita College of Engineering and Technology, \\ Amritagiri, Erachakulam Post, Nagercoil-629902, Tamil Nadu, India \\ rajmath84@gmail.com
}

S. Saravanakumar

Kalasalingam Academy of Research and Education, Anand Nagar, Krishnankoil-626126, Tamil Nadu, India

alg.ssk@gmail.com

\begin{abstract}
Let $G$ be a graph with the vertex set $V(G)$. A subset $S$ of $V(G)$ is an open packing set of $G$ if every pair of vertices in $S$ has no common neighbor in $G$. The maximum cardinality of an open packing set of $G$ is the open packing number of $G$ and it is denoted by $\rho^{o}(G)$. In this paper, the exact values of the open packing numbers for some classes of perfect graphs, such as split graphs, $\left\{P_{4}, C_{4}\right\}$-free graphs, the complement of a bipartite graph, the trestled graph of a perfect graph are obtained.
\end{abstract}

Keywords: Open packing number, 2-packing number, Perfect graphs, Trestled graphs.

\section{Introduction}

By a graph $G=(V, E)$, we mean a finite, undirected graph with neither loops nor multiple edges. For graph theoretic terminology, we refer to the book by Chartrand and Lesniak [4]. For a vertex $v$ in $V(G)$, the open neighborhood of $v$ and the closed neighborhood of $v$ are defined by $N(v)=\{u \in V(G): u v \in E(G)\}$ and $N[v]=N(v) \cup\{v\}$, respectively. Similarly for a subset $S$ of $V(G)$, the open and closed neighborhoods of $S$ are defined by $N(S)=\cup_{v \in S} N(v)$ and $N[S]=\cup_{v \in S} N[v]$. For any two sets $A$ and $B$, define $A \backslash B=\{x: x \in A$ and $x \notin B\}$. In a graph $G$, a vertex of degree 1 is a leaf and the vertex adjacent to a leaf is a support vertex of $G$. For a set $S$ of vertices of $G$, the induced subgraph is the maximal subgraph of $G$ with vertex set $S$ and is denoted by $\langle S\rangle$. Thus two vertices of $S$ are adjacent in $\langle S\rangle$ if and only if they are adjacent in $G$.

A subset $S$ of $V(G)$ is independent if no two vertices in $S$ are adjacent in $G$. The independence number $\beta_{0}(G)$ is the maximum cardinality of an independent set in $G$. A subset $M$ of $E(G)$ is independent if no two edges in $M$ are adjacent in $G$. A set of independent edges in $G$ is a matching of $G$. The edge independence number $\beta_{1}(G)$ is the maximum cardinality of a matching in $G$.

A proper coloring of a graph $G$ is an assignment of colors to the vertices of $G$ in such a way that no two adjacent vertices receive the same color. The chromatic number $\chi(G)$ is the minimum number of colors required for a proper coloring of $G$. A clique in $G$ is a complete subgraph of $G$. The maximum order of a clique in $G$ is the clique number of $G$ and is denoted by $\omega(G)$. A graph $G$ is perfect if $\chi(H)=\omega(H)$ for every induced subgraph $H$ of $G$.

Perfect graphs were introduced by Berge [2], who conjectured that a graph $G$ is perfect if and only if $\bar{G}$ is perfect. This Perfect Graph Conjecture was verified by Lovász [10]. Since the chromatic number and clique number of an odd cycle of length at least 5 are not equal, it follows that if an 
induced subgraph of a graph $G$ is an odd cycle of length at least 5 , then the graphs $G$ and $\bar{G}$ are not perfect. Berge [3] conjectured that every graph that is not perfect contains either an induced odd cycle of length at least 5 or its complement graph $\bar{G}$ contains such a cycle. This famous conjecture (sometimes referred to as the Strong Perfect Graph Conjecture) was verified by Chudnovsky et al. [5]. It is well known that $\chi(G) \geq \omega(G)$, for every graph $G$. Hence if $G$ contains a triangle, then $\chi(G) \geq 3$. It may be surprising that Mycielski [13] has proved that there exist triangle free graphs with large chromatic number. Several classes of perfect graphs and their properties are given in [9].

A subset $S$ of $V(G)$ is an open packing set of $G$ if every pair of vertices in $S$ has no common neighbor in $G$. The maximum cardinality of an open packing set of $G$ is the open packing number of $G$ and is denoted by $\rho^{o}(G)$. An open packing set of cardinality $\rho^{o}(G)$ is a $\rho^{o}$-set of $G$. Clearly every open packing set contains at most one vertex in every open neighborhood $N(v)$ of a vertex $v$ in $G$. A subset $S^{\prime}$ of $V(G)$ is a 2-packing set of $G$ if every pair of vertices in $S^{\prime}$ is of distance at least 3 in $G$. The maximum cardinality of a 2-packing set is the 2-packing number and is denoted by $\rho(G)$. We observe that for any connected graph $G$, every 2-packing set of $G$ is an open packing set of $G$ and hence $\rho(G) \leq \rho^{o}(G)$.

The concepts of 2-packing and open packing sets in graphs were introduced respectively by Meir and Moon [11] and Henning et.al [8]. Fisher et al. [7] proved that for any connected graph $G$, $\rho(G)=\rho(\mu(G))$, where $\mu(G)$ is the Mycielskian of the graph $G$. Henning and Slater [8] proved that for any graph $G$ of order $n \rho^{o}(G) \leq 2 n / 3$. They also obtained the characterization of all graphs $G$ for which $\rho^{o}(G)=2 n / 3$. In [14], the authors have proved that $\rho^{o}(G) \leq n / \delta(G)$. Moreover, the characterization of all connected graphs for which the equality holds was settled in [12].

In this paper, we obtain the exact values of the open packing number for some families of perfect graphs, such as, split graphs, $\left\{P_{4}, C_{4}\right\}$-free graphs, the complement of a bipartite graph and the trestled graph of a perfect graph.

\section{Split graphs and $\left\{\mathrm{P}_{4}, \mathrm{C}_{4}\right\}$-free graphs}

Definition 1. A graph $G$ is a $\left\{P_{4}, C_{4}\right\}$-free graph if neither $P_{4}$ nor $C_{4}$ is an induced subgraph of $G$.

We know that every $\left\{P_{4}, C_{4}\right\}$-free graph is a perfect graph, see [15]. We now determine the exact value of the open packing number for $\left\{P_{4}, C_{4}\right\}$-free graphs. The following assertion is used to prove the theorem.

Assertion 1. Let $S$ be an open packing set of $G$. Then every component of the induced subgraph $\langle S\rangle$ is isomorphic to either $K_{1}$ or $K_{2}$.

Theorem 1. Let $G$ be a $\left\{P_{4}, C_{4}\right\}$-free graph of order $n \geq 2$. Then $\rho^{\circ}(G)$ is either 1 or 2 . Further, $\rho^{o}(G)=2$ if and only if $\Delta(G)=n-1$ and $\delta(G)=1$.

P r o o f. If $\operatorname{diam}(G) \geq 3$, then $G$ contains a $P_{4}$ as an induced subgraph, which is a contradiction. Hence $\operatorname{diam}(G)$ is either 1 or 2 . If $\operatorname{diam}(G)=1$, then $G$ is a complete graph $K_{n}$ and hence $\rho^{o}(G)$ is either 1 or 2 depending on whether $n \geq 3$ or $n=2$.

Suppose $\operatorname{diam}(G)=2$. Let $S$ be a $\rho^{o}$-set of $G$. If $|S| \geq 3$, then by Assertion 1 there exist two vertices $u$ and $v$ in $G$ such that $u, v \in S$ and $u v \notin E(\langle S\rangle)$. Consequently $u v \notin E(G)$. Since $\operatorname{diam}(G)=2$, there exists a vertex $x$ in $V(G)$ such that $x$ is adjacent to $u$ and $v$ in $G$, which is a contradiction to $u$ and $v$ are in a $\rho^{o}$-set $S$ of $G$. Hence $\rho^{o}(G)$ is either 1 or 2 .

We claim that $\rho^{o}(G)=2$ if and only if $\Delta(G)=n-1$ and $\delta(G)=1$. Suppose $\rho^{o}(G)=2$ and let $S^{\prime}=\{u, v\}$ be a $\rho^{o}$-set of $G$. It follows from the above argument that $u v \in E(G)$. 
Let $D_{1}=N_{G}(u) \backslash\{v\}$ and $D_{2}=N_{G}(v) \backslash\{u\}$. Since $u$ and $v$ have no common neighbor in $G$, it follows that $D_{1} \cap D_{2}=\emptyset$.

If $D_{1}=D_{2}=\emptyset$, then $G=K_{2}$, and we are done. Hence $D_{1} \neq \emptyset$ or $D_{2} \neq \emptyset$. Suppose $D_{1} \neq \emptyset$ and $D_{2} \neq \emptyset$ and let $x \in D_{1}$ and $y \in D_{2}$. Then $u y \notin E(G)$ and $v x \notin E(G)$ and hence the induced subgraph $\langle\{u, v, x, y\}\rangle$ is isomorphic to either $P_{4}$ or $C_{4}$ depending on $x y \notin E(G)$ or $x y \in E(G)$, which is a contradiction to $G$ is a $\left\{P_{4}, C_{4}\right\}$-free graph. Hence we may assume without loss of generality that $D_{1} \neq \emptyset$ and $D_{2}=\emptyset$. Then $\operatorname{deg}(v)=1$ and $u$ is the support vertex adjacent to $v$ in $G$.

Now we claim that $V(G)=N[u]$. If $z \in V(G) \backslash N[u]$, then $d(z, v) \geq 3$, which is a contradiction to $\operatorname{diam}(G) \leq 2$. Hence $V(G)=N[u]$. It follows that $\operatorname{deg}(u)=n-1$ and $\operatorname{deg}(v)=1$. Thus $\Delta(G)=n-1$ and $\delta(G)=1$.

Conversely, suppose $\Delta(G)=n-1$ and $\delta(G)=1$. Let $x, y \in V(G)$ be such that $\operatorname{deg}(x)=n-1$ and $\operatorname{deg}(y)=1$. Clearly $S=\{x, y\}$ is an open packing set of $G$ and hence $\rho^{o}(G) \geq|S|=2$. Moreover, any open packing set of $G$ contains at most one vertex in $N(x)=V(G) \backslash\{x\}$, it follows that $\rho^{o}(G)=2$.

Definition 2. A split graph $G$ is a graph whose vertex set can be partitioned into two sets $K$ and $I$, where the vertices in $K$ form a complete graph and the vertices in $I$ are independent. The partition $(K, I)$ is a split partition of the split graph $G$.

Clearly every split graph is a perfect graph, see [9]. We now determine the open packing number of a split graph $G$ in terms of the 2-packing number $\rho(G)$.

Theorem 2. Let $G$ be a connected split graph of order $n$ with split partition $(K, I)$ and $\Delta(G)<n-1$. Then $\rho^{o}(G)=\rho(G)$.

P r o o f. Let $S$ be a $\rho^{o}$-set of the split graph $G$. Since no two vertices in $S$ have a common neighbor in $G$ and the induced subgraph $\langle K\rangle$ is complete, it follows that $|S \cap K| \leq 2$. We consider the following three cases depending on $|S \cap K|$ is 0,1 , or 2 .

Case i. $|S \cap K|=0$.

Then $S \subseteq I$ and hence the distance between any pair of vertices in $S$ is exactly 3. Consequently $S$ is a 2-packing set of $G$ and hence $\rho^{o}(G)=|S \cap I| \leq \rho(G)$. Thus $\rho^{o}(G)=\rho(G)$ as $\rho(G) \leq \rho^{o}(G)$.

Case ii. $|S \cap K|=1$.

Let $S \cap K=\{u\}$. Now we claim that $|S|$ is either 2 or 1 depending on whether $u$ is a support vertex of $G$ or not. Let $v^{\prime} \in I$ be such that $v^{\prime} u \notin E(G)$, the existence of $v^{\prime}$ is guaranteed by the assumption that $\Delta(G)<n-1$. Let $v$ be a neighbor of $v^{\prime}$ in $K$. Then $v^{\prime} \notin S$, otherwise $u$ and $v^{\prime}$ in $S$ have a common neighbor, namely $v$, in $G$, which is a contradiction. Further, $N(u) \cap I$ contains at most one vertex of $S$, it follows that $|S \cap I|$ is either 0 or 1 . If $|S \cap I|=0$, then $|S|=1$ and hence $S$ is a 2-packing set of $G$. Consequently $\rho^{o}(G)=\rho(G)=1$.

Suppose $|S \cap I|=1$ and let $S \cap I=\left\{u^{\prime}\right\}$. Then $u^{\prime}$ must be a leaf neighbor of $u$ in $G$, otherwise $u$ and $u^{\prime}$ will have a common neighbor in $G$, which is a contradiction. Hence $S=\left\{u, u^{\prime}\right\}$, where $\operatorname{deg}\left(u^{\prime}\right)=1$ and $u u^{\prime} \in E(G)$. Now consider the set $S^{\prime}=(S \backslash\{u\}) \cup\left\{v^{\prime}\right\}$, where $v^{\prime} \notin N(u)$. Clearly $S^{\prime}$ is a 2-packing set of $G$ and hence $\left|S^{\prime}\right|=2=\rho^{o}(G) \leq \rho(G)$. Thus $\rho(G)=\rho^{o}(G)$.

Case iii. $|S \cap K|=2$.

Let $S \cap K=\{u, v\}$. We claim that $|S \cap I|=0$. Suppose $z^{\prime} \in S \cap I$ and let $z$ be a neighbor of $z^{\prime}$ in $K$. If $z=u$, then $z^{\prime}$ and $v$ in $S$ have a common neighbor, namely $u$, in $G$, which is a contradiction. Similarly, if $z=v$, then $z^{\prime}$ and $u$ in $S$ have a common neighbor in $G$, which is a contradiction. Finally, suppose $z \notin\{u, v\}$. Then $z^{\prime}$ and $u$ have a common neighbor, namely $z$, in $G$, which is again a contradiction. Thus $|S \cap I|=0$. Since $G$ is a connected split graph and $\Delta(G)<n-1$, it follows that $\operatorname{diam}(G)=3$. Let $u^{\prime}, v^{\prime} \in V(G)$ be such that $d\left(u^{\prime}, v^{\prime}\right)=3$. Then 
$\left\{u^{\prime}, v^{\prime}\right\} \subseteq I$ and the set $S^{\prime}=\left\{u^{\prime}, v^{\prime}\right\}$ is a 2-packing set of $G$. Hence $2=\rho^{o}(G) \leq \rho(G)$. Thus $\rho^{o}(G)=\rho(G)$.

Remark 1. If $G$ is a split graph of order $n$ with $\Delta(G)=n-1$, then $\rho(G)=1$ and

$$
\rho^{o}(G)= \begin{cases}2 & \text { if } \delta(G)=1 \\ 1 & \text { otherwise }\end{cases}
$$

\section{Complement of a bipartite graph and the trestled graph of a graph}

In this section, we determine the exact values of the open packing number of the complement of a bipartite graph and the trestled graph of a graph.

Theorem 3. Let $G$ be a connected bipartite graph which is not a complete bipartite graph with bipartition $(X, Y)$ and $2 \leq|X| \leq|Y|$. Then $\rho^{o}(\bar{G})$ is either 1 or 2 . Further, $\rho^{o}(\bar{G})=2$ if and only if either $|X|=2$ or, there exist two vertices $x \in X$ and $y \in Y$ such that $N_{G}(x) \supseteq Y \backslash\{y\}$ and $N_{G}(y) \supseteq X \backslash\{x\}$.

P r o o f. We first claim that $\rho^{o}(\bar{G})$ is either 1 or 2 . Since $G$ is not a complete bipartite graph, let $x \in X$ and $y \in Y$ be such that $x y \notin E(G)$. Then $x y \in E(\bar{G})$.

Let $N_{x}=(X \backslash\{x\}) \cup\{y\}$ and $N_{y}=(Y \backslash\{y\}) \cup\{x\}$. Clearly $N_{x} \subseteq N_{\bar{G}}(x), N_{y} \subseteq N_{\bar{G}}(y)$ and $N_{x} \cup N_{y}=V(\bar{G})$ as $x y \in E(\bar{G})$ and, $\langle X\rangle$ and $\langle Y\rangle$ are complete subgraphs in $\bar{G}$. Let $S$ be a $\rho^{o}$-set in $\bar{G}$. Then $\left|S \cap N_{\bar{G}}(x)\right| \leq 1$ and $\left|S \cap N_{\bar{G}}(y)\right| \leq 1$. Hence $\left|S \cap N_{x}\right| \leq 1$ and $\left|S \cap N_{y}\right| \leq 1$ as $N_{x} \subseteq N_{\bar{G}}(x)$ and $N_{y} \subseteq N_{\bar{G}}(y)$. Consequently

$$
\left|S \cap\left(N_{x} \cup N_{y}\right)\right|=|S \cap V(\bar{G})|=|S|=\rho^{o}(\bar{G}) \leq 2 .
$$

Thus $\rho^{o}(\bar{G})$ is either 1 or 2 .

Suppose $\rho^{o}(\bar{G})=2$. Let $S_{1}=\left\{u^{\prime}, v^{\prime}\right\}$ be a $\rho^{o}$-set of $\bar{G}$. Now we consider the following three cases depending on $u^{\prime}$ and $v^{\prime}$ are in $X$ or, $Y$ or, $X$ and $Y$, respectively, in $\bar{G}$.

Case i. $u^{\prime}, v^{\prime} \in X$.

If $|X| \geq 3$, then there exists a vertex, say $w^{\prime}$, in $X$ such that $w^{\prime}$ is adjacent to $u^{\prime}$ and $v^{\prime}$ in $\bar{G}$, which is a contradiction. Suppose $|X|=2$. Then $X=\left\{u^{\prime}, v^{\prime}\right\}$. If there exists a vertex, say $z^{\prime}$, in $Y$ such that $z^{\prime}$ is adjacent to $u^{\prime}$ and $v^{\prime}$ in $\bar{G}$, then $z^{\prime}$ is an isolated vertex, a vertex of degree 0 , in $G$ and hence $G$ is disconnected, which is a contradiction. Thus every vertex of $Y$ is adjacent to at most one vertex in $X$. Consequently $X$ is an open packing set in $\bar{G}$ and hence $\rho^{o}(\bar{G})=|X|=2$.

Case ii. $u^{\prime}, v^{\prime} \in Y$.

It follows from the similar argument of Case i that $\rho^{o}(\bar{G})=|Y|=2$. Since $2 \leq|X| \leq|Y|=$ $\rho^{o}(\bar{G})=2$, it follows that $\rho^{o}(\bar{G})=|X|=2$.

Case iii. $u^{\prime} \in X$ and $v^{\prime} \in Y$.

If $N_{\bar{G}}\left(u^{\prime}\right) \cap\left(Y \backslash\left\{v^{\prime}\right\}\right) \neq \emptyset$ or $N_{\bar{G}}\left(v^{\prime}\right) \cap\left(X \backslash\left\{u^{\prime}\right\}\right) \neq \emptyset$, then there exists a vertex in $\bar{G}$ which is commonly adjacent to $u^{\prime}$ and $v^{\prime}$, which is a contradiction to $S_{1}=\left\{u^{\prime}, v^{\prime}\right\}$ is an open packing set in $\bar{G}$. Hence $N_{\bar{G}}\left(u^{\prime}\right) \cap\left(Y \backslash\left\{v^{\prime}\right\}\right)=\emptyset$ and $N_{\bar{G}}\left(v^{\prime}\right) \cap\left(X \backslash\left\{u^{\prime}\right\}\right)=\emptyset$. Consequently $N_{G}\left(u^{\prime}\right) \supseteq Y \backslash\left\{v^{\prime}\right\}$ and $N_{G}\left(v^{\prime}\right) \supseteq X \backslash\left\{u^{\prime}\right\}$.

The converse is obvious.

Remark 2. If $G$ is a complete bipartite graph $K_{m, n}, 2 \leq m \leq n$, then

$$
\rho^{o}(\bar{G})=\rho^{o}\left(K_{m} \cup K_{n}\right)= \begin{cases}4 & \text { if } m=n=2, \\ 3 & \text { if } m=2 \text { and } n \geq 3, \\ 2 & \text { otherwise. }\end{cases}
$$


Definition 3. Let $G=(V, E)$ be a graph and let $k$ be any positive integer. The trestled graph $T_{k}(G)$ of index $k$ is the graph obtained from $G$ by adding $k$ copies of $K_{2}$ to each edge $u v$ of $G$ and joining $u$ and $v$ to the respective end vertices of each $K_{2}$.

The trestled graph $T_{2}\left(C_{5}\right)$ is given in Fig. 1. Some basic algorithmic results on trestled graphs are given in [1] and [6]. Clearly $\chi\left(T_{k}(G)\right)=\chi(G)$ and $\omega\left(T_{k}(G)\right)=\omega(G)$ and hence if $G$ is a perfect graph, then $T_{k}(G)$ is also a perfect graph.

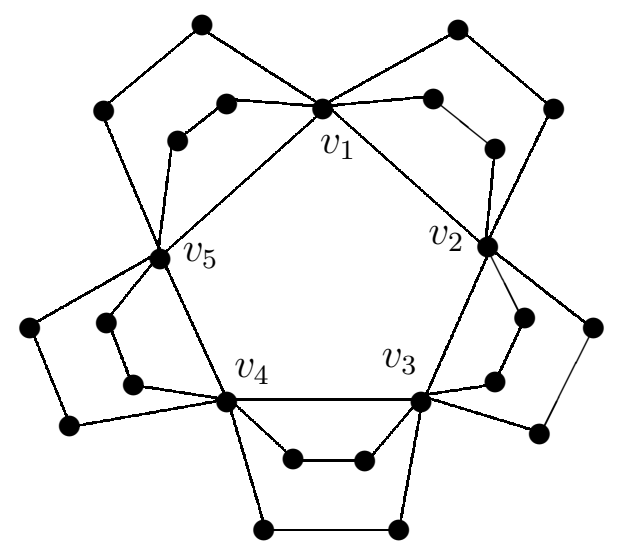

Figure 1. The trestled graph $T_{2}\left(C_{5}\right)$

Theorem 4. Let $G$ be a connected graph of order $n$ and let $k$ be any positive integer. Then $\rho^{o}\left(T_{k}(G)\right)=n$.

P r o o f. Let $V(G)=\left\{v_{1}, v_{2}, \ldots, v_{n}\right\}$. Let $N_{1}=N_{G}\left(v_{1}\right)$ and, for every $i, 2 \leq i \leq n$, $N_{i}=N_{G}\left(v_{i}\right) \backslash\left(\bigcup_{j=1}^{i-1} N_{G}\left(v_{j}\right)\right)$. Clearly $N_{1}, N_{2}, \ldots, N_{n}$ are disjoint, $\bigcup_{i=1}^{n} N_{i}=V(G)$ and, for every $i, 1 \leq i \leq n, N_{i} \subseteq N_{G}\left(v_{i}\right)$. Now we consider the partition of $V\left(T_{k}(G)\right) \backslash V(G)$ into $n$ disjoint subsets, say $N_{1}^{\prime}, N_{2}^{\prime}, \ldots, N_{n}^{\prime}$, as follows: for every $i, 1 \leq i \leq n$, let $N_{i}^{\prime}=N_{T_{k}(G)}\left(v_{i}\right) \backslash N_{G}\left(v_{i}\right)$. Clearly $V\left(T_{k}(G)\right)=\bigcup_{i=1}^{n}\left(N_{i} \cup N_{i}^{\prime}\right)$ and, for every $i, 1 \leq i \leq n, N_{i} \cup N_{i}^{\prime} \subseteq N_{T_{k}(G)}\left(v_{i}\right)$.

Now we claim that $\rho^{o}\left(T_{k}(G)\right)=n$. Let $S$ be a $\rho^{o}$-set of $T_{k}(G)$. Then for every $i, 1 \leq$ $i \leq n,\left|S \cap N_{T_{k}(G)}\left(v_{i}\right)\right| \leq 1$ and hence $\left|S \cap\left(N_{i} \cup N_{i}^{\prime}\right)\right| \leq 1$ as $N_{i} \cup N_{i}^{\prime} \subseteq N_{T_{k}(G)}\left(v_{i}\right)$. Since $V\left(T_{k}(G)\right)=\bigcup_{i=1}^{n}\left(N_{i} \cup N_{i}^{\prime}\right)$, it follows that $\rho^{o}\left(T_{k}(G)\right)=|S| \leq n$. Also for each $i, 1 \leq i \leq n$, choose $x_{i} \in N_{i}^{\prime}$. Clearly $S^{\prime}=\left\{x_{1}, x_{2}, \ldots, x_{n}\right\}$ is an open packing set of $T_{k}(G)$ as no two $x_{i}^{\prime s}$ have a common neighbor in $T_{k}(G)$. Hence $\rho^{o}\left(T_{k}(G)\right) \geq n$. Thus $\rho^{o}\left(T_{k}(G)\right)=n$.

\section{Conclusion and scope}

In this paper we have determined the exact value of open packing number for some families of perfect graphs. Designing efficient algorithms for computing $\rho^{o}(G)$ for other classes of perfect graphs such as, interval graphs, circular arc graphs, bipartite graphs are some interesting problems for further investigation. In particular, finding the open packing number for trees is a challenging open problem for further study.

\section{Acknowledgements}

The authors thank the referees for their valuable comments and suggestions leading to the present form of this paper. 


\section{REFERENCES}

1. Aparna Lakshmanan S., Vijayakumar A. The $\langle t\rangle$-property of some classes of graphs. Discrete Math., 2009. Vol. 309, No. 1. P. 259-263. DOI: 10.1016/j.disc.2007.12.057

2. Berge C. The Theory of Graphs and its Applications. London: Methuen, 1962. 257 p.

3. Berge C. Graphs and Hypergraphs. London: North-Holland, 1973. 528 p.

4. Chartrand G., Lesniak L. Graphs and Digraphs. $4^{\text {th }}$ ed. London: Chapman and Hall/CRC, 2005. 386 p.

5. Chudnovsky M., Robertson N., Seymour P., Thomas R. The strong perfect graph theorem. Ann. Math., 2006. Vol. 164, No. 1. P. 51-229. DOI: 10.4007/annals.2006.164.51

6. Fellows M., Fricke G., Hedetniemi S., Jacobs D. The private neighbor cube. SIAM J. Discrete Math., 1994. Vol. 7, No. 1. P. 41-47. DOI: 10.1137/S0895480191199026

7. Fisher D. C., Mckenna P.A., Boyer E.D. Hamiltonicity, diameter, domination, packing and biclique partitions of Mycielski's graphs. Discrete Appl. Math., 1998. Vol. 84, No. 1-3. P. 93-105. DOI: 10.1016/S0166-218X(97)00126-1

8. Henning M. A., Slater P. J. Open packing in graphs. J. Combin. Math. Combin. Comput., 1999. Vol. 29. P. 3-16.

9. Hougardy S. Classes of perfect graphs. Discrete Math., 2006. Vol. 306, No. 19-20. P. 2529-2571. DOI: $10.1016 /$ j.disc.2006.05.021

10. Lovász L. Normal hypergraphs and the perfect graph conjecture. Discrete Math., 1972. Vol. 2, No. 3. P. 253-267. DOI: 10.1016/0012-365X(72)90006-4

11. Meir A., Moon J. W. Relations between packing and covering numbers of a tree. Pacific J. Math., 1975. Vol. 61, No. 1. P. 225-233. https://projecteuclid.org/euclid.pjm/1102868240

12. Mojdeh D.A., Samadi B., Khodkar A. and Golmohammadi H.R. On the packing numbers in graphs. Australas. J. Combin., 2018. Vol. 71, No. 3. P. 468-475. https://ajc.maths.uq.edu.au/pdf/71/ajc_v71_p468.pdf

13. Mycielski J. Sur le coloriage des graphes. Colloq. Math., 1955. Vol. 3. P. 161-162.

14. Sahul Hamid I., Saravanakumar S. Packing parameters in graphs. Discuss. Math. Graph Theory, 2015. Vol. 35. P. 5-16. DOI: $10.7151 /$ dmgt. 1775

15. Seinsche D. On a property of the class of $n$-colorable graphs. J. Combin. Theory Ser. B, 1974. Vol. 16, No. 2. P. 191-193. DOI: 10.1016/0095-8956(74)90063-X 